\title{
High Accuracy Computation of Rank-Constrained Fundamental Matrix
}

\author{
Yasuyuki Sugaya \\ Department of Information and Computer Sciences, \\ Toyohashi University of Technology, Toyohashi, Aichi 441-8580 Japan \\ sugaya@iim.ics.tut.ac.jp \\ Kenichi Kanatani \\ Department of Computer Science, Okayama University, \\ Okayama 700-8530 Japan \\ kanatani@suri.it.okayama-u.ac.jp
}

\begin{abstract}
A new method is presented for computing the fundamental matrix from point correspondences: its singular value decomposition (SVD) is optimized by the Levenberg-Marquard (LM) method. The search is initialized by optimal correction of unconstrained ML. There is no need for tentative 3-D reconstruction. The accuracy achieves the theoretical bound (the KCR lower bound).
\end{abstract}

\section{Introduction}

Computing the fundamental matrix from point correspondences is the first step of many vision applications including camera calibration, image rectification, structure from motion, and new view generation [7].

A popular approach is to do maximum likelihood (ML) computation without imposing the constraint that the fundamental matrix has rank 2; the rank constraint was imposed a posteriori in a statistically optimal manner. The resulting solution has accuracy close to the theoretical bound (KCR lower bound $[2,8]$ ).

Another approach is to do optimization subject to the rank constraint $[1,4,13,14]$. In this paper, we propose a new method in this line. Following Bartoli and Sturm [1], we optimize the singular value decomposition (SVD) of the fundamental matrix, but there is no need to include 3-D coordinates or camera matrices as unknowns; we use the LevenbergMarquard (LM) method in the reduced parameter space.

We numerically compare our LM approach with the optimal correction and show that they have complementary properties. The optimal correction is fairly accurate and robust but gradually deteriorates as noise increases. In contrast, LM achieves very high accuracy when started from a good initial value but is likely to fall into local minima if poorly initialized. From this, we conclude that starting LM from the optimal correction achieves the highest accuracy, which agrees with the theoretical bound (the KCR lower bound). The result is also compared with the bundle adjustment approach. 
We summarize the mathematical background in Sect. 2. We describe the optimal correction approach in Sect. 3 and our LM approach in Sect. 4. In Sect. 5, we discuss the bundle adjustment in relation to our methods. Sect. 6 shows numerical experiments. We conclude in Sect. 7.

\section{Mathematical Background}

Fundamental matrix. Given two images of the same scene, a point $(x, y)$ in the first image and the corresponding point $\left(x^{\prime}, y^{\prime}\right)$ in the second satisfy the epipolar equation [7]

$$
\left(\left(\begin{array}{c}
x \\
y \\
f_{0}
\end{array}\right),\left(\begin{array}{lll}
F_{11} & F_{12} & F_{13} \\
F_{21} & F_{22} & F_{23} \\
F_{31} & F_{32} & F_{33}
\end{array}\right)\left(\begin{array}{c}
x^{\prime} \\
y^{\prime} \\
f_{0}
\end{array}\right)\right)=0,
$$

where the image origin $(0,0)$ is at the frame center and $f_{0}$ is a scaling constant of the order of the image size (this is for stabilizing numerical computation [6]. In our experiments, we set $f_{0}=600$ pixels). Throughout this paper, we denote the inner product of vectors a and $\mathbf{b}$ by $(\mathbf{a}, \mathbf{b})$. The matrix $\mathbf{F}=\left(F_{i j}\right)$ in Eq. (1) is of rank 2 and called the fundamental matrix. If we define

$$
\begin{aligned}
& \mathbf{u}=\left(F_{11}, F_{12}, F_{13}, F_{21}, F_{22}, F_{23}, F_{31}, F_{32}, F_{33}\right)^{\top}, \\
& \xi=\left(x x^{\prime}, x y^{\prime}, x f_{0}, y x^{\prime}, y y^{\prime}, y f_{0}, f_{0} x^{\prime}, f_{0} y^{\prime}, f_{0}^{2}\right)^{\top},
\end{aligned}
$$

Equation (1) can be rewritten as

$$
(\mathbf{u}, \xi)=0 .
$$

The magnitude of $\mathbf{u}$ is indeterminate, so we normalize it to $\|\mathbf{u}\|=1$. If we write $N$ observed noisy correspondence pairs as 9-D vectors $\left\{\xi_{\alpha}\right\}$ in the form of Eq. (3), our task is to estimate the 9-D vector $\mathbf{u}$ from $\left\{\xi_{\alpha}\right\}$ using Eq. (4).

Covariance matrices. We write $\xi_{\alpha}=\bar{\xi}_{\alpha}+\Delta \xi_{\alpha}$, where $\bar{\xi}_{\alpha}$ is the true value and $\Delta \xi_{\alpha}$ the noise term. The covariance matrix of $\xi_{\alpha}$ is defined by

$$
V\left[\xi_{\alpha}\right]=E\left[\Delta \xi_{\alpha} \Delta \xi_{\alpha}^{\top}\right]
$$

where $E[\cdot]$ denotes expectation over the noise distribution. If the noise in the $x$ - and $y$ coordinates is independent and of mean 0 and standard deviation $\sigma$, the covariance matrix of $\xi_{\alpha}$ has the form $V\left[\xi_{\alpha}\right]=\sigma^{2} V_{0}\left[\xi_{\alpha}\right]$ up to $O\left(\sigma^{4}\right)$, where

$$
V_{0}\left[\xi_{\alpha}\right]=\left(\begin{array}{ccccccccc}
\bar{x}_{\alpha}^{2}+\bar{x}_{\alpha}^{\prime 2} & \bar{x}_{\alpha}^{\prime} \bar{y}_{\alpha}^{\prime} & f_{0} \bar{x}_{\alpha}^{\prime} & \bar{x}_{\alpha} \bar{y}_{\alpha} & 0 & 0 & f_{0} \bar{x}_{\alpha} & 0 & 0 \\
\bar{x}_{\alpha}^{\prime} \bar{y}_{\alpha}^{\prime} & \bar{x}_{\alpha}^{2}+\bar{y}_{\alpha}^{\prime 2} & f_{0} \bar{y}_{\alpha}^{\prime} & 0 & \bar{x}_{\alpha} \bar{y}_{\alpha} & 0 & 0 & f_{0} \bar{x}_{\alpha} & 0 \\
f_{0} \bar{x}_{\alpha}^{\prime} & f_{0} \bar{y}_{\alpha}^{\prime} & f_{0}^{2} & 0 & 0 & 0 & 0 & 0 & 0 \\
\bar{x}_{\alpha} \bar{y}_{\alpha} & 0 & 0 & \bar{y}_{\alpha}^{2}+\bar{x}_{\alpha}^{\prime 2} & \bar{x}_{\alpha}^{\prime} \bar{y}_{\alpha}^{\prime} & f_{0} \bar{x}_{\alpha}^{\prime} & f_{0} \bar{y}_{\alpha} & 0 & 0 \\
0 & \bar{x}_{\alpha} \bar{y}_{\alpha} & 0 & \bar{x}_{\alpha}^{\prime} \bar{y}_{\alpha}^{\prime} & \bar{y}_{\alpha}^{2}+\bar{y}_{\alpha}^{\prime 2} & f_{0} \bar{y}_{\alpha}^{\prime} & 0 & f_{0} \bar{y}_{\alpha} & 0 \\
0 & 0 & 0 & f_{0} \bar{x}_{\alpha}^{\prime} & f_{0} \bar{y}_{\alpha}^{\prime} & f_{0}^{2} & 0 & 0 & 0 \\
f_{0} \bar{x}_{\alpha} & 0 & 0 & f_{0} \bar{y}_{\alpha} & 0 & 0 & f_{0}^{2} & 0 & 0 \\
0 & f_{0} \bar{x}_{\alpha} & 0 & 0 & f_{0} \bar{y}_{\alpha} & 0 & 0 & f_{0}^{2} & 0 \\
0 & 0 & 0 & 0 & 0 & 0 & 0 & 0 & 0
\end{array}\right) .
$$


In actual computations, we replace the true positions $\left(\bar{x}_{\alpha}, \bar{y}_{\alpha}\right)$ and $\left(\bar{x}_{\alpha}^{\prime}, \bar{y}_{\alpha}^{\prime}\right)$ by their data $\left(x_{\alpha}, y_{\alpha}\right)$ and $\left(x_{\alpha}^{\prime}, y_{\alpha}^{\prime}\right)$, respectively (experiments have confirmed that this does not cause noticeable changes in final results).

We define the covariance matrix $V[\hat{\mathbf{u}}]$ of the resulting estimate $\hat{\mathbf{u}}$ of $\mathbf{u}$ by

$$
V[\hat{\mathbf{u}}]=E\left[\left(\mathbf{P}_{\mathscr{U}} \hat{\mathbf{u}}\right)\left(\mathbf{P}_{\mathscr{U}} \hat{\mathbf{u}}\right)^{\top}\right]
$$

where $\mathbf{P}_{\mathscr{U}}$ is the linear operator projecting $\mathscr{R}^{9}$ onto the domain $\mathscr{U}$ of $\mathbf{u}$ defined by the constraints $\|\mathbf{u}\|=1$ and $\operatorname{det} \mathbf{F}=0$; we evaluate the error of $\hat{\mathbf{u}}$ by projecting it onto the tangent space $T_{\mathbf{u}}(\mathscr{U})$ to $\mathscr{U}$ at $\mathbf{u}$.

Geometry of the constraint. The normal to the hypersurface $\operatorname{defined}$ by $\operatorname{det} \mathbf{F}=0$ is $\nabla_{\mathbf{u}} \operatorname{det} \mathbf{F}$. After scale normalization, it is written as

$$
\mathbf{u}^{\dagger} \equiv N\left[\left(\begin{array}{c}
u_{5} u_{9}-u_{8} u_{6} \\
u_{6} u_{7}-u_{9} u_{4} \\
u_{4} u_{8}-u_{7} u_{5} \\
u_{8} u_{3}-u_{2} u_{9} \\
u_{9} u_{1}-u_{3} u_{7} \\
u_{7} u_{2}-u_{1} u_{8} \\
u_{2} u_{6}-u_{5} u_{3} \\
u_{3} u_{4}-u_{6} u_{1} \\
u_{1} u_{5}-u_{4} u_{2}
\end{array}\right)\right]
$$

where $N[\cdot]$ denotes normalization into unit norm (this vector represents the "cofactor" of F). It is easily seen that the constraint $\operatorname{det} \mathbf{F}=0$ is equivalently written as

$$
\left(\mathbf{u}^{\dagger}, \mathbf{u}\right)=0 .
$$

Since the domain $\mathscr{U}$ is included in the unit sphere $\mathscr{S}^{8} \subset \mathscr{R}^{9}$, the vector $\mathbf{u}$ is everywhere orthogonal to $\mathscr{U}$. Hence, $\left\{\mathbf{u}, \mathbf{u}^{\dagger}\right\}$ is an orthonormal basis of the orthogonal complement of the tangent space $T_{\mathbf{u}}(\mathscr{U})$. It follows that the projection operator $\mathbf{P}_{\mathscr{U}}$ in Eq. (7) has the following matrix representation (I is the unit matrix):

$$
\mathbf{P}_{\mathscr{U}}=\mathbf{I}-\mathbf{u} \mathbf{u}^{\top}-\mathbf{u}^{\dagger} \mathbf{u}^{\dagger \top} .
$$

KCR lower bound. If the noise in $\left\{\xi_{\alpha}\right\}$ is independent and Gaussian with mean $\mathbf{0}$ and covariance matrix $\sigma^{2} V_{0}\left[\xi_{\alpha}\right]$, the following inequality holds for an arbitrary unbiased estimator $\hat{\mathbf{u}}$ of $\mathbf{u}[8]$ :

$$
V[\hat{\mathbf{u}}] \succ \sigma^{2}\left(\sum_{\alpha=1}^{N} \frac{\left(\mathbf{P}_{\mathscr{U}} \bar{\xi}_{\alpha}\right)\left(\mathbf{P}_{\mathscr{U}} \bar{\xi}_{\alpha}\right)^{\top}}{\left(\mathbf{u}, V_{0}\left[\xi_{\alpha}\right] \mathbf{u}\right)}\right)_{8}^{-} .
$$

Here, $\succ$ means that the left-hand side minus the right is positive semidefinite, and $(\cdot)_{r}^{-}$ denotes the pseudoinverse of rank $r$. Chernov and Lesort [2] called the right-hand side of Eq. (11) the KCR (Kanatani-Cramer-Rao) lower bound and showed that Eq. (11) holds up to $O\left(\sigma^{4}\right)$ even if $\hat{\mathbf{u}}$ is not unbiased; it is sufficient that $\hat{\mathbf{u}} \rightarrow \mathbf{u}$ as $\sigma \rightarrow 0$.

Maximum likelihood. If the noise in $\left\{\xi_{\alpha}\right\}$ is independent and Gaussian with mean $\mathbf{0}$ and covariance matrix $\sigma^{2} V_{0}\left[\xi_{\alpha}\right]$, maximum likelihood (ML) estimation of $\mathbf{u}$ is to minimize the 
sum of square Mahalanobis distances

$$
J=\sum_{\alpha=1}^{N}\left(\xi_{\alpha}-\bar{\xi}_{\alpha}, V_{0}\left[\xi_{\alpha}\right]_{2}^{-}\left(\xi_{\alpha}-\bar{\xi}_{\alpha}\right)\right),
$$

subject to $\left(\mathbf{u}, \bar{\xi}_{\alpha}\right)=0, \alpha=1, \ldots, N$. Eliminating the constraint by using Lagrange multipliers, we obtain [8]

$$
J=\sum_{\alpha=1}^{N} \frac{\left(\mathbf{u}, \xi_{\alpha}\right)^{2}}{\left(\mathbf{u}, V_{0}\left[\xi_{\alpha}\right] \mathbf{u}\right)} .
$$

The ML estimator $\hat{\mathbf{u}}$ minimizes this subject to $\|\mathbf{u}\|=1$ and $\left(\mathbf{u}^{\dagger}, \mathbf{u}\right)=0$.

\section{Optimal Correction}

A common approach to solve this problem is to minimizes Eq. (13) without considering the rank constraint, compute the SVD of the resulting fundamental matrix, and replace the smallest singular value by 0 , producing a "closest" matrix of rank 2 in norm [6]. We call this SVD correction.

A more sophisticated method is the optimal correction $[8,12]$. According to the statistical optimization theory [8], the covariance matrix $V[\tilde{\mathbf{u}}]$ of the rank unconstrained solution $\tilde{\mathbf{u}}$ can be evaluated, so $\tilde{\mathbf{u}}$ is moved in the direction of the mostly likely fluctuation implied by $V[\tilde{\mathbf{u}}]$ until it satisfies the rank constraint. The procedure goes as follows [8]:

1. Compute the following $9 \times 9$ matrix $\tilde{\mathbf{M}}$ :

$$
\tilde{\mathbf{M}}=\sum_{\alpha=1}^{N} \frac{\xi_{\alpha} \xi_{\alpha}^{\top}}{\left(\tilde{\mathbf{u}}, V_{0}\left[\xi_{\alpha}\right] \tilde{\mathbf{u}}\right)} .
$$

2. Compute the normalized covariance matrix $V_{0}[\tilde{\mathbf{u}}]$ as follows:

$$
V_{0}[\tilde{\mathbf{u}}]=\tilde{\mathbf{M}}_{8}^{-}
$$

3. Update the solution $\tilde{\mathbf{u}}$ as follows ( $\tilde{\mathbf{u}}^{\dagger}$ is defined by Eq. (8) for $\left.\tilde{\mathbf{u}}\right)$ :

$$
\tilde{\mathbf{u}} \leftarrow N\left[\tilde{\mathbf{u}}-\frac{1}{3} \frac{\left(\tilde{\mathbf{u}}, \tilde{\mathbf{u}}^{\dagger}\right) V_{0}[\tilde{\mathbf{u}}] \tilde{\mathbf{u}}^{\dagger}}{\left(\tilde{\mathbf{u}}^{\dagger}, V_{0}[\tilde{\mathbf{u}}] \tilde{\mathbf{u}}^{\dagger}\right)}\right] .
$$

4. If $\left(\tilde{\mathbf{u}}, \tilde{\mathbf{u}}^{\dagger}\right) \approx 0$, return $\tilde{\mathbf{u}}$ and stop. Else, update the normalized covariance matrix $V_{0}[\tilde{\mathbf{u}}]$ in the form

$$
\mathbf{P}_{\tilde{\mathbf{u}}}=\mathbf{I}-\tilde{\mathbf{u}} \tilde{\mathbf{u}}^{\top}, \quad V_{0}[\tilde{\mathbf{u}}] \leftarrow \mathbf{P}_{\tilde{\mathbf{u}}} V_{0}[\tilde{\mathbf{u}}] \mathbf{P}_{\tilde{\mathbf{u}}},
$$

and go back to Step 3 .

Before doing this, we need to solve unconstrained minimization of Eq. (13), for which several methods exist: the FNS (Fundamental Numerical Scheme) of Chojnacki et al. [3], the HEIV (Heteroscedastic Errors-in-Variable) of Leedan and Meer [11], and the projective Gauss-Newton iterations of Kanatani and Sugaya [9]. Their convergence properties were studies in [9]. 


\section{Direct Search}

We now propose a new method for minimizing Eq. (13) subject to the rank constraint. The fundamental matrix $\mathbf{F}$ has nine elements, on which the normalization $\|\mathbf{F}\|=1$ and the rank constraint $\operatorname{det} \mathbf{F}=0$ are imposed. So, it has seven degrees of freedom.

Many types of 7-degree parameterization have been proposed in the past. Typical ones are based on epipoles (e.g., $[13,14]$ ), but the resulting expressions are often complicated, and the geometric meaning of the individual unknowns are not clear. This was overcome by Bartoli and Sturm [1], who regarded the SVD of $\mathbf{F}$ as its parameterization. Their expression is compact, and each parameter has its geometric meaning. However, they included, in addition to $\mathbf{F}$, the 3 -D positions of the observed feature points, the relative positions of the two cameras, and their intrinsic parameters as unknowns. Using assumed values, they computed tentative 3 -D reconstruction and evaluated the reprojection error. Then, they searched the high-dimensional parameter space for the value that minimizes the reprojection error. Since the tentative 3-D reconstruction from two images is indeterminate, they chose the one for which the first camera matrix is in a particular form ("canonical form").

From the underlying geometry, however, we can see that the necessary and sufficient condition for the corresponding points $\left\{\left(x_{\alpha}, y_{\alpha}\right)\right\}$ and $\left\{\left(x_{\alpha}^{\prime}, y_{\alpha}^{\prime}\right)\right\}$ to be a projection of "some" 3-D structure is the epipolar equation of Eq. (1) for "some" F, and Eq. (12) describes the corresponding reprojection error. Hence, bundle adjustment for minimizing the reprojection error by assuming "some" 3-D reconstruction is practically equivalent to minimize Eq. (12) (the meaning of "practically" is discussed shortly), so we need not consider indeterminate $3-\mathrm{D}$ reconstruction.

Here, using the parameterization of Bartoli and Sturm [1], we directly minimize Eq. (13) by the Levenberg-Marquard (LM) method. The fundamental matrix $\mathbf{F}$ has rank 2, so its SVD has the form

$$
\mathbf{F}=\mathbf{U d i a g}\left(\sigma_{1}, \sigma_{2}, 0\right) \mathbf{V}^{\top},
$$

where $\mathbf{U}$ and $\mathbf{V}$ are orthogonal matrices, and $\sigma_{1}$ and $\sigma_{2}$ are the singular values. Since the normalization $\|\mathbf{F}\|^{2}=1$ is equivalent to $\sigma_{1}^{2}+\sigma_{2}^{2}=1$, we adopt the following parameterization $^{1}$ :

$$
\sigma_{1}=\cos \theta, \quad \sigma_{2}=\sin \theta .
$$

The orthogonal matrices $\mathbf{U}$ and $\mathbf{V}$ have three degrees of freedom each, so they and $\theta$ constitute the seven degrees of freedom. However, the analysis becomes complicated if $\mathbf{U}$ and $\mathbf{V}$ are directly expressed in three parameters each (e.g., the Euler angles or the rotations around each coordinate axis). Following Bartoli and Sturm [1], we adopt the "Lie algebraic method": we represent the "increment" in $\mathbf{U}$ and $\mathbf{V}$ by three parameters each. Let $\omega_{1}, \omega_{2}$, and $\omega_{3}$ represent the increment in $\mathbf{U}$, and $\omega_{1}^{\prime}, \omega_{2}^{\prime}$, and $\omega_{3}^{\prime}$ in $\mathbf{V}$. The derivatives of Eq. (13) with respect to them are as follows (we omit the details):

$$
\nabla_{\omega} J=\mathbf{F}_{U}^{\top} \mathbf{X u}, \quad \nabla_{\omega^{\prime}} J=\mathbf{F}_{V}^{\top} \mathbf{X u} .
$$

\footnotetext{
${ }^{1}$ Bartoli and Sturm [1] took the ratio $\gamma=\sigma_{2} / \sigma_{1}$ as a variable. Here, we adopt the angle $\theta$ for the symmetry. As is well known, it has the value $\pi / 4$ (i.e., $\sigma_{1}=\sigma_{2}$ ) if the principal point is at the origin $(0,0)$ and if there are no image distortions $[7,8]$.
} 
Here, the matrices $\mathbf{X}, \mathbf{F}_{U}$, and $\mathbf{F}_{V}$ are defined by

$$
\begin{gathered}
\mathbf{X}=\sum_{\alpha=1}^{N} \frac{\xi_{\alpha} \xi_{\alpha}^{\top}}{\left(\mathbf{u}, V_{0}\left[\xi_{\alpha}\right] \mathbf{u}\right)}-\sum_{\alpha=1}^{N} \frac{\left(\mathbf{u}, \xi_{\alpha}\right)^{2} V_{0}\left[\xi_{\alpha}\right]}{\left(\mathbf{u}, V_{0}\left[\xi_{\alpha}\right] \mathbf{u}\right)^{2}}, \\
\mathbf{F}_{U}=\left(\begin{array}{rrr}
0 & F_{31} & -F_{21} \\
0 & F_{32} & -F_{22} \\
0 & F_{33} & -F_{23} \\
-F_{31} & 0 & F_{11} \\
-F_{32} & 0 & F_{12} \\
-F_{33} & 0 & F_{13} \\
F_{21} & -F_{11} & 0 \\
F_{22} & -F_{12} & 0 \\
F_{23} & -F_{13} & 0
\end{array}\right), \quad \mathbf{F}_{V}=\left(\begin{array}{rrr}
0 & F_{13} & -F_{12} \\
-F_{13} & 0 & F_{11} \\
F_{12} & -F_{11} & 0 \\
0 & F_{23} & -F_{22} \\
-F_{23} & 0 & F_{21} \\
F_{22} & -F_{21} & 0 \\
0 & F_{33} & -F_{32} \\
-F_{33} & 0 & F_{31} \\
F_{32} & -F_{31} & 0
\end{array}\right) .
\end{gathered}
$$

The derivative of Eq. (13) with respect to $\theta$ is

$$
\frac{\partial J}{\partial \theta}=\left(\mathbf{u}_{\theta}, \mathbf{X u}\right),
$$

where we define

$$
\mathbf{u}_{\theta}=\left(\begin{array}{l}
U_{12} V_{12} \cos \theta-U_{11} V_{11} \sin \theta \\
U_{12} V_{22} \cos \theta-U_{11} V_{21} \sin \theta \\
U_{12} V_{32} \cos \theta-U_{11} V_{31} \sin \theta \\
U_{22} V_{12} \cos \theta-U_{21} V_{11} \sin \theta \\
U_{22} V_{22} \cos \theta-U_{21} V_{21} \sin \theta \\
U_{22} V_{32} \cos \theta-U_{21} V_{31} \sin \theta \\
U_{32} V_{12} \cos \theta-U_{31} V_{11} \sin \theta \\
U_{32} V_{22} \cos \theta-U_{31} V_{21} \sin \theta \\
U_{32} V_{32} \cos \theta-U_{31} V_{31} \sin \theta
\end{array}\right) .
$$

Adopting Gauss-Newton approximation, which amounts to ignoring terms involving $\left(\mathbf{u}, \xi_{\alpha}\right)$, we obtain the second derivatives as follows (we omit the details):

$$
\begin{array}{ccc}
\nabla_{\omega}^{2} J=\mathbf{F}_{U}^{\top} \mathbf{X F}_{U}, & \nabla_{\omega^{\prime}}^{2} J=\mathbf{F}_{V}^{\top} \mathbf{X} \mathbf{F}_{V}, & \nabla_{\omega \omega^{\prime}} J=\mathbf{F}_{U}^{\top} \mathbf{X F}_{V}, \\
\frac{\partial J^{2}}{\partial \theta^{2}}=\left(\mathbf{u}_{\theta}, \mathbf{X u}_{\theta}\right), & \frac{\partial \nabla_{\omega} J}{\partial \theta}=\mathbf{F}_{U}^{\top} \mathbf{X u}_{\theta}, & \frac{\partial \nabla_{\omega^{\prime}} J}{\partial \theta}=\mathbf{F}_{V}^{\top} \mathbf{X} \mathbf{u}_{\theta} .
\end{array}
$$

The LM procedure goes as follows:

1. Initialize $\mathbf{F}$ in such a way that $\operatorname{det} \mathbf{F}=0$ and $\|\mathbf{F}\|=1$, and express it as $\mathbf{F}=$ $\mathbf{U d i a g}(\cos \theta, \sin \theta, 0) \mathbf{V}^{\top}$.

2. Compute $J$ in Eq. (13), and let $c=0.0001$.

3. Compute $\mathbf{F}_{U}, \mathbf{F}_{V}$, and $\mathbf{u}_{\theta}$ in Eqs. (22) and (24).

4. Compute $\mathbf{X}$ in Eq. (21), the first derivatives in Eqs. (20) an (23), and the second derivatives in Eqs. (25).

5. Compute the following matrix $\mathbf{H}$ :

$$
\mathbf{H}=\left(\begin{array}{ccc}
\nabla_{\omega}^{2} J & \nabla_{\omega \omega^{\prime}} J & \partial \nabla_{\omega} J / \partial \theta \\
\left(\nabla_{\omega \omega^{\prime}} J\right)^{\top} & \nabla_{\omega^{\prime}}^{2} J & \partial \nabla_{\omega^{\prime}} J / \partial \theta \\
\left(\partial \nabla_{\omega} J / \partial \theta\right)^{\top} & \left(\partial \nabla_{\omega^{\prime}} J / \partial \theta\right)^{\top} & \partial J^{2} / \partial \theta^{2}
\end{array}\right) .
$$


6. Solve the 7-D simultaneous linear equations

$$
(\mathbf{H}+c D[\mathbf{H}])\left(\begin{array}{c}
\omega \\
\omega^{\prime} \\
\Delta \theta
\end{array}\right)=-\left(\begin{array}{c}
\nabla_{\omega} J \\
\nabla_{\omega^{\prime}} J \\
\partial J / \partial \theta
\end{array}\right),
$$

for $\omega, \omega^{\prime}$, and $\Delta \theta$, where $D[\cdot]$ denotes the diagonal matrix obtained by taking out only the diagonal elements.

7. Update $\mathbf{U}, \mathbf{V}$, and $\theta$ by

$$
\mathbf{U}^{\prime}=\mathscr{R}(\omega) \mathbf{U}, \quad \mathbf{V}^{\prime}=\mathscr{R}\left(\omega^{\prime}\right) \mathbf{V}, \quad \theta^{\prime}=\theta+\Delta \theta,
$$

where $\mathscr{R}(\omega)$ denotes rotation around $N[\omega]$ by angle $\|\omega\|$.

8. Update $\mathbf{F}$ as follows:

$$
\mathbf{F}^{\prime}=\mathbf{U}^{\prime} \operatorname{diag}\left(\cos \theta^{\prime}, \sin \theta^{\prime}, 0\right) \mathbf{V}^{\prime \top}
$$

9. Let $J^{\prime}$ be the value of Eq. (13) for $\mathbf{F}^{\prime}$.

10. Unless $J^{\prime}<J$ or $J^{\prime} \approx J$, let $c \leftarrow 10 c$, and go back to Step 6 .

11. If $\mathbf{F}^{\prime} \approx \mathbf{F}$, return $\mathbf{F}^{\prime}$ and stop. Else, let $\mathbf{F} \leftarrow \mathbf{F}^{\prime}, \mathbf{U} \leftarrow \mathbf{U}^{\prime}, \mathbf{V} \leftarrow \mathbf{V}^{\prime}, \theta \leftarrow \theta^{\prime}$, and $c \leftarrow$ $c / 10$, and go back to Step 3 .

\section{Bundle Adjustment}

In the discussion of Sect. 2, there is a subtle point to be clarified. The transition from Eq. (12) to Eq. (13) is exact; no approximation is involved. Although terms of $O\left(\sigma^{4}\right)$ are omitted and the true values are replaced by their data in Eq. (6), it is numerically confirmed that these do not affect the final results in any noticeable way.

However, although the "analysis" may be exact, the "interpretation" is not strict. Namely, despite the fact that Eq. (13) is the (squared) Mahalanobis distance in the $\xi$ space, its minimization can be ML only when the noise in the $\xi$-space is Gaussian, because then and only then is the likelihood proportional to $e^{-J / \text { constant }}$. Strictly speaking, if the noise in the image plane is Gaussian, the transformed noise in the $\xi$-space is no longer Gaussian, so minimizing Eq. (13) is not strictly ML in the image plane.

In order to test how much difference is incurred, we implemented the bundle adjustment in the image plane (we omit the details) and did comparison experiments.

\section{Experiments}

Figure 1 shows simulated images of two planar grid surfaces viewed from different angles. The image size is $600 \times 600$ pixels with 1200 pixel focal length. We added random Gaussian noise of mean 0 and standard deviation $\sigma$ to the $x$ - and $y$-coordinates of each grid point independently and from them computed the fundamental matrix by 1) SVDcorrected LS, 2) SVD-corrected ML, 3) CFNS, 4) optimally corrected ML, and 5) LM.

"LS" means least squares (also called "eight-point algorithm" [6]) minimizing $\sum_{\alpha=1}^{N}\left(\mathbf{u}, \xi_{\alpha}\right)^{2}$, which reduces to simple eigenvalue computation [9]. For brevity, we use the shorthand "ML" for unconstrained minimization of Eq. (13), for which we used the 

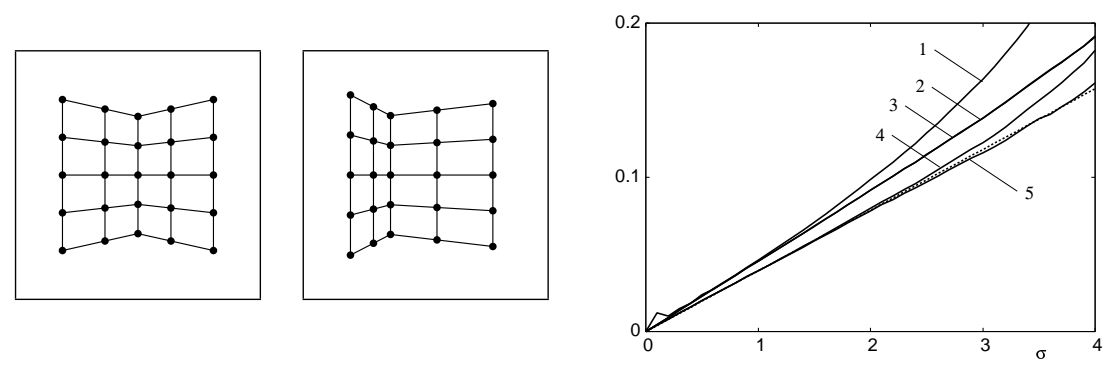

Figure 1: Simulated images of planar grid surfaces and the RMS error vs. noise level. 1) SVDcorrected LS. 2) SVD-corrected ML. 3) CFNS, 4) Optimally corrected ML 5) LM. The dotted line indicates the KCR lower bound.

FNS of Chojnacki et al. [3]. "CFNS" [4] computes the rank-constrained fundamental matrix by repeated eigenvalue computation. The LM and CFNS are initialized by LS. All iterations are stopped when the update of $\mathbf{F}$ is less than $10^{-6}$ in norm.

On the right Fig. 1 is plotted for $\sigma$ on the horizontal axis the following root-meansquare (RMS) error $D$ corresponding to Eq. (7) over 10000 independent trials:

$$
D=\sqrt{\frac{1}{10000} \sum_{a=1}^{10000}\left\|\mathbf{P}_{\mathscr{U}} \hat{\mathbf{u}}^{(a)}\right\|^{2}}
$$

Here, $\hat{\mathbf{u}}^{(a)}$ is the $a$ th value, and $\mathbf{P}_{\mathscr{U}}$ is the projection matrix in Eq. (10). The dotted line is the bound implied by the KCR lower bound (the trace of the right-hand side of Eq. (11)).

Preliminary observations. We can see that the most popular SVD-corrected LS [6] performs very poorly. We can also see that SVD-corrected ML is inferior to optimally corrected ML $[8,12]$, whose accuracy is close to the KCR lower bound. The accuracy of LM is nearly the same as optimally corrected ML when the noise is small but gradually outperforms it as the noise increases.

Doing many experiments (not all shown here), we have observed that i) optimally corrected ML is fairly accurate and very robust to noise but gradually deteriorates as noise increases, and ii) LM achieves very high accuracy when started from a good initial value but is likely to fall into local minima if poorly initialized.

The robustness of optimally corrected ML is due to the fact that the computation is done in the redundant ("external") $\mathbf{u}$-space, where $J$ has a simple form of Eq. (13). In fact, we have never experienced local minima in our experiments. The deterioration in the presence of large noise is because linear approximation is involved in Eq. (16).

The fragility of LM is attributed to the complexity of the function $J$ when expressed in seven parameters, resulting in many local minima in the reduced ("internal") parameter space, as pointed out in [13].

Thus, we conclude that the best result is obtained by LM initialized by optimally corrected ML. The CFNS of Chojnacki et al. [4] performs as poorly as SVD-corrected ML. They asserted superiority of CFNS to optimally corrected ML by numerical examples [4], but Fig. 1 contradicts their assertion. The reason for this is fully investigated in [10], where a new method called EFNS is proposed to complement CFNS. So, we do not go into the details here. 


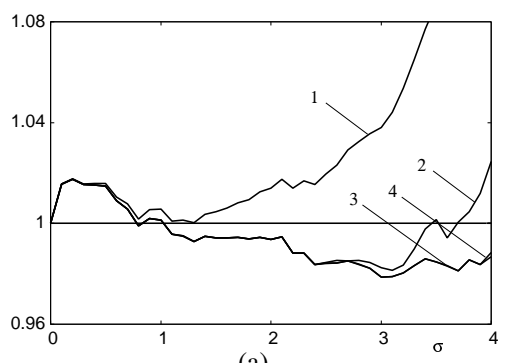

(a)

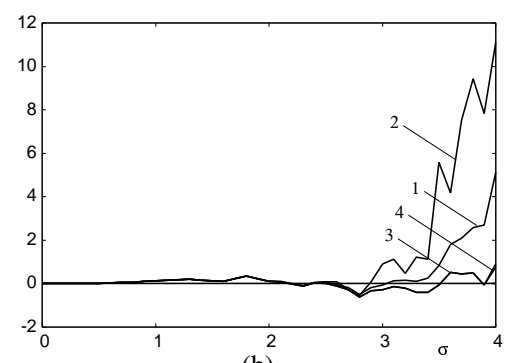

(b)

Figure 2: (a) The RMS error relative to the KCR lower bound. (b) The average residual minus $(N-7) \sigma^{2}$. 1) Optimally corrected ML. 2) LM from LS. 3) LM from optimally corrected ML. 4) Bundle adjustment in the image plane.
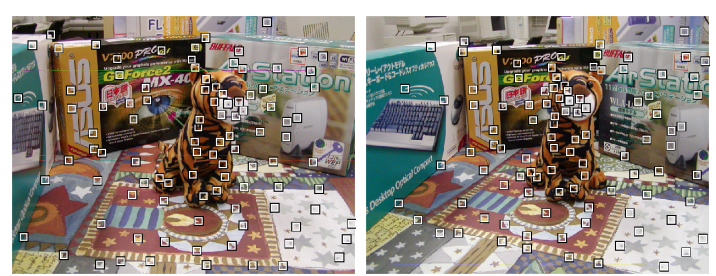

\begin{tabular}{l|r|c} 
& residual & time \\
\hline \hline 1$)$ & 45.550 & .00052 \\
\hline 2$)$ & 45.556 & .00652 \\
\hline 3$)$ & 45.556 & .01300 \\
\hline 4$)$ & 45.378 & .00764 \\
\hline 5$)$ & 45.378 & .01136 \\
\hline 6$)$ & 45.378 & .01748 \\
\hline 7$)$ & 45.378 & .02580
\end{tabular}

Figure 3: Left: Real images and 100 corresponding points. Right: Residuals and execution times (sec) for 1) SVD-corrected LS, 2) SVD-corrected ML, 3) CFNS, 4) optimally corrected ML, 5) LM from LS, 6) LM from optimally corrected ML, 7) bundle adjustment in the image plane.

Detailed observations. Figure 2(a) compares 1) optimally corrected ML, 2) LM initialized by LS, 3) LM initialized by optimally corrected ML, and 4) bundle adjustment in the image plane (see Sect. 5). Since the error plots almost coincide if displayed as in Fig. 1, we plot here the ratio $D / D_{\mathrm{KCR}}$ of $D$ in Eq. (30) to the corresponding KCR lower bound. Figure 2(b) shows the corresponding residual $J$ (minimum of Eq. (13)). For visual ease, we plot its difference from $(N-7) \sigma^{2}$, where $N$ is the number of corresponding pairs $\left(J / \sigma^{2}\right.$ is to a first approximation subject to a $\chi^{2}$ distribution with $N-7$ degrees of freedom [8], so the expectation of $J$ is approximately $\left.(N-7) \sigma^{2}\right)$.

We can observe that i) the RMS error of optimally corrected ML increases as noise increases, yet the corresponding residual remains low, meaning that Eq. (13) is very "flat", having nearly the same value around its minimum, ii) LM from LS appears to have high accuracy for noise levels for which the corresponding residual is high, indicating that the solution falls into local minima nearer to the true value, iii) the accuracy of LM improves if initialized by optimally corrected ML, and iv) the result agrees with bundle adjustment in the image plane.

Real image example. Figure 3 shows real images, from which we manually selected 100 pairs of corresponding points and computed the fundamental matrix from them. Since the true value is unknown, we evaluated the final residual $J$ together with the execution time (sec) as listed there. We used Core2Duo E6700 2.66GHz for the CPU with 4GB main memory and Linux for the OS. As expected, SVD-corrected ML is very poor. Again, CFNS performs no better than SVD-corrected ML. Optimally corrected ML and LM (from LS or from optimally corrected ML) arrive at the same solution, which also results from bundle adjustment with a much longer computation time. 


\section{Conclusions}

We presented a new method for computing the fundamental matrix from point correspondences over two images ${ }^{2}$ : we adopted the SVD representation of Bartoli and Sturm [1] and optimized it by LM. There is no need for tentative 3-D reconstruction. We concluded that our LM method initialized by optimally corrected ML achieves the accuracy comparable to the KCR lower bound and much time-consuming bundle adjustment.

Acknowledgments: The authors thank Wojciech Chojnacki of the University of Adelaide, Australia for providing

\section{References}

[1] A. Bartoli and P. Sturm, Nonlinear estimation of fundamental matrix with minimal parameters, IEEE Trans. Pattern Anal. Mach. Intell., 26-3 (2004-3), 426-432.

[2] N. Chernov and C. Lesort, Statistical efficiency of curve fitting algorithms, Comput. Stat. Data Anal., 47-4 (2004-11), 713-728.

[3] W. Chojnacki, M. J. Brooks, A. van den Hengel and D. Gawley, On the fitting of surfaces to data with covariances, IEEE Trans. Pattern Anal. Mach. Intell., 22-11 (2000-11), 1294-1303.

[4] W. Chojnacki, M. J. Brooks, A. van den Hengel and D. Gawley, A new constrained parameter estimator for computer vision applications Image Vis. Comput., 22-2 (2004-2), 85-91.

[5] W. Chojnacki, M. J. Brooks, A. van den Hengel and D. Gawley, FNS, CFNS and HEIV: A unifying approach, J. Math. Imaging Vision, 23-2 (2005-9), 175-183.

[6] R. I. Hartley, In defense of the eight-point algorithm, IEEE Trans. Patt. Anal. Mach. Intell., 19-6 (1997-6), 580-593.

[7] R. Hartley and A. Zisserman, Multiple View Geometry in Computer Vision, Cambridge University Press, Cambridge, U.K., 2000.

[8] K. Kanatani, Statistical Optimization for Geometric Computation: Theory and Practice, Elsevier Science, Amsterdam, The Netherlands, 1996; Dover, New York, 2005.

[9] K. Kanatani and Y. Sugaya, High accuracy fundamental matrix computation and its performance evaluation, Proc. 17th British Machine Vision Conf., September 2006, Edinburgh, U.K., Vol. 1, pp. 217-226.

[10] K. Kanatani and Y. Sugaya, Extended FNS for constrained parameter estimation, Proc. 10th Meeting Image Recog. Understand, Hiroshima, Japan, July 2007.

[11] Y. Leedan and P. Meer, Heteroscedastic regression in computer vision: Problems with bilinear constraint, Int. J. Comput. Vision., 37-2 (2000-6), 127-150.

[12] J. Matei and P. Meer, Estimation of nonlinear errors-in-variables models for computer vision applications, IEEE Trans. Patt. Anal. Mach. Intell., 28-10 (2006-10), 1537-1552.

[13] T. Migita and T. Shakunaga, One-dimensional search for reliable epipole estimation, Proc. IEEE Pacific Rim Symp. Image and Video Technology, December 2006, Hsinchu, Taiwan, pp. 1215-1224.

[14] Z. Zhang and C. Loop, Estimating the fundamental matrix by transforming image points in projective space, Comput. Vis. Image Understand., 82-2 (2001), 174-180.

\footnotetext{
${ }^{2}$ The source code is available at http://www.iim.ics.tut.ac.jp/ sugaya/public-e.html
} 